\title{
NEW PARALLELOGRAM 3D-DISPLACEMENT SENSOR FOR MICRO PROBING AND DIMENTIONAL METROLOGY
}

\author{
David Metz $^{l}$ and Andreas Dietzel ${ }^{1}$ \\ ${ }^{1}$ Technische Universität Braunschweig, Braunschweig, GERMANY
}

\begin{abstract}
For the use in dimensional metrology a novel micro probing system composed of three silicon parallelogram linkages was developed. The parallelogram structure is assured by elastic silicon hinge and allows displacement in only one direction. For each parallelogram the displacement is evaluate using piezo-resistors integrated in the silicon hinges. Wide deflection ranges, isotropic behavior and low mechanical stiffness as predicted by simulations could be verified in experiments. With this combination of characteristics the micro probing system is an ideal sensor for the use in all conventional CMMs but also for many other 3D force/displacement measurement tasks.
\end{abstract}

\section{KEYWORDS}

3D microprobe, isotropy, big deflection range, silicon-parallelogram, piezo-resistor, tactile measurement

\section{INTRODUCTION}

A variety of micro probing systems has been developed for the measurement of micro geometries in particular on the base of silicon material [1-3]. Such probing systems are known for small deflection ranges and for high anisotropies, resulting from their construction of silicon membranes with integrated sensing elements on which a stylus is attached [1-3]. Work has been dedicated to get isotropic mechanics, but could not be achieved without increasing the stiffnesses, which is not desirable in micro measurements [4, 5]. For such microprobes, deflection ranges do typically not exceed $\pm 100 \mu \mathrm{m}$ [2], which is not suitable for an easy integration in commercial CMM or in other measurement setups, because of a too high risk of system damage.

The new 3D micro probing system (3DSP) presented in this work is composed of three identical silicon parallelograms (SP) assembled in cascade (s. Figure 1a.). The parallelogram is composed of three bonded silicon parts (s. fabrication process). To build the parallelogram structure elastic hinges made of a thin silicon membrane are used. Each of them allows a deflection in only one direction over a larger range (s. Figure 1b.). To be able to measure each deflection for each direction, piezo-resistors are integrated in the silicon membrane. Processing and bonding of the SP has been developed on wafer level to enable cost effective fabrication at larger quantities.

As a first step in the development process, mechanical simulations of a single SP and of the complete microprobe were undertaken. This served as basis for the definition of geometrical dimensions of the SP. In a next step, the fabrication process was developed, which will be explained in detail. Finally the obtained first prototypes were experimentally characterized.

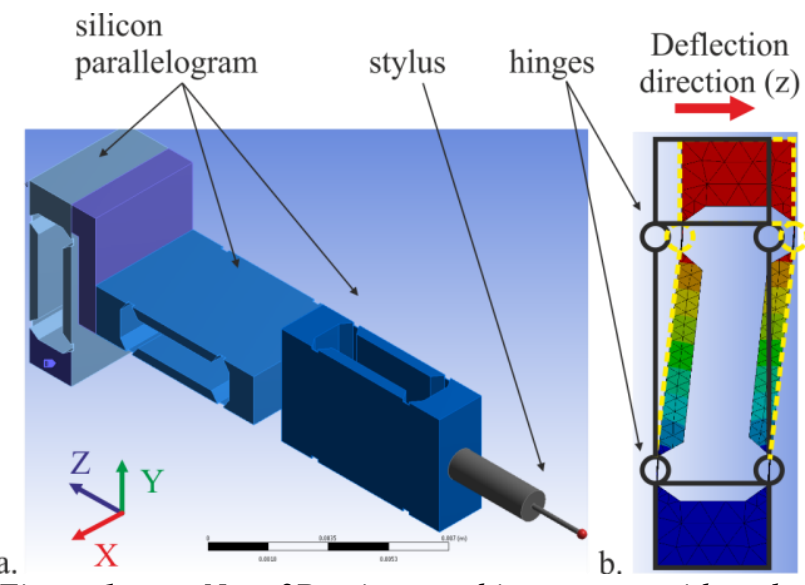

Figure $1-a$. New 3D micro probing system with stylus and $b$. simulation/principle of the deflection of a single silicon parallelogram

\section{SIMULATION}

To predict and to confirm the operation of this new system, mechanical simulations were carried out in Ansys 16.0. In Figure 2, the simulations results are presented. Forces $(50 \mathrm{mN})$ in the threes directions were applied to the 3DSP and a deflection of $60 \mu \mathrm{m}$ in the direction of the applied force were obtained. In each three orthogonal directions only one of the three identical SP deflected. This showed an isotropic behavior of the simulated system with a low stiffness $\left(<1 \mathrm{mN} . \mu \mathrm{m}^{-1}\right)$.
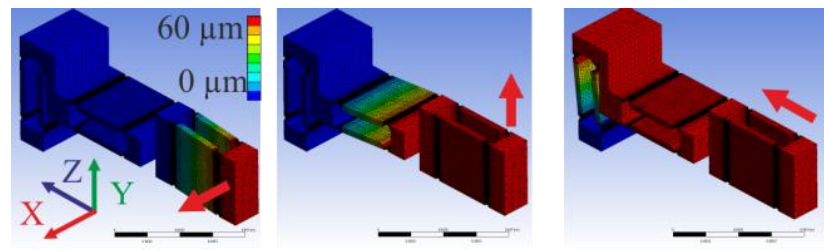

Figure 2 - Deflection of a 3DSP for a force of $50 \mathrm{mN}$ force in $x$-, $y$ - and $z$-direction as obtained by simulations

In an ideal case the single SP deflects in only the main direction $\mathrm{z}$ (s. Figure 1b. and Figure 3). In practice, the thin hinges may deformed slightly also in other directions ( $\mathrm{x}$ and $\mathrm{y}$ ), which can cause measurement uncertainties. So that these deformations are considered negligible, a minimum ration between the stiffness in zdirection and $\mathrm{x}$ - or $\mathrm{y}$-directions as to be assured. The ratio $\mathrm{S}_{\mathrm{x}} / \mathrm{S}_{\mathrm{z}}$ or $\mathrm{S}_{\mathrm{y}} / \mathrm{S}_{\mathrm{z}}$ has to be bigger than 1000 , to assure an uncertainty in sub-micron range. In this purpose, the single SP was simulated in order to optimize its geometrical dimensions. Therefore the dimensions $\mathrm{W}_{\mathrm{mem}}$, $\mathrm{t}_{\mathrm{mem}}, \mathrm{L}_{\mathrm{mem}}$ (see Figure 3) were parametrized in order to carry out parameter variation studies in Ansys. 


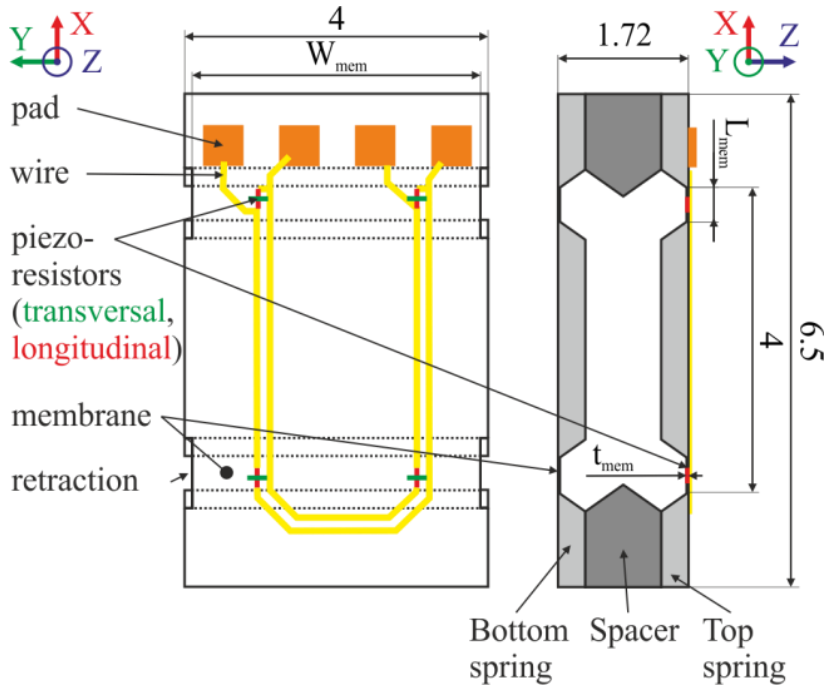

Figure 3 - Single parallelogram construction and definition of dimensional parameters

These three parameters define the geometry of the membrane, which is used as hinge, and all other dimensions were kept constant. For the simulation one side of the SP was fixed and a force $(50 \mathrm{mN})$ was applied in the three directions $(\mathrm{x}, \mathrm{y}$ and $\mathrm{z}$ ) on the other side. In combination with the calculated deflections, corresponding stiffnesses $\left(S_{x}, S_{y}\right.$ and $\left.S_{z}\right)$ were analyzed.

In Figure 4 the stiffness $S_{x}$ and $S_{z}$ are presented as function of $\mathrm{L}_{\text {mem }}$ and $\mathrm{t}_{\mathrm{mem}}$ for a $\mathrm{W}_{\text {mem }}=3.8 \mathrm{~mm}$. The stiffness $S_{y}$ is not represented, because it behaves as $S_{x}$. The simulations confirm an expected general mechanical trend: with a longer, slimmer and/or a thinner membrane the stiffness decreases.
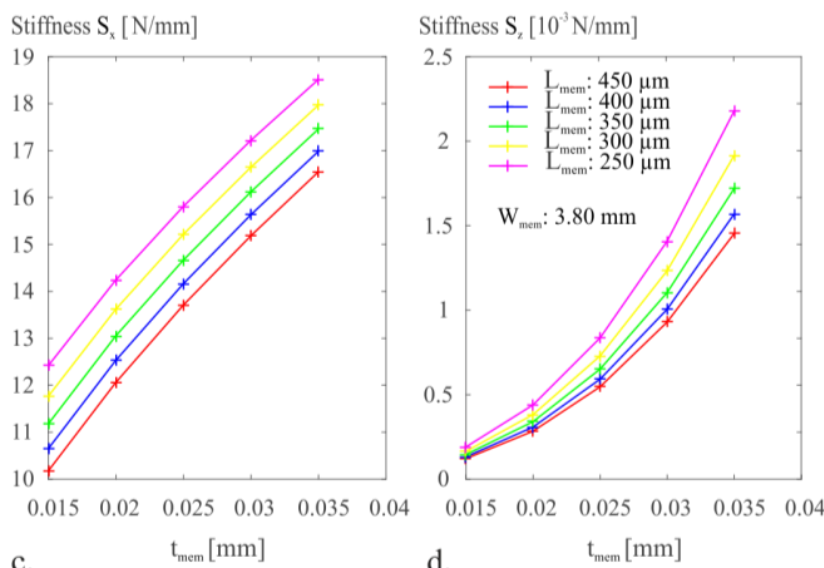

Figure 4 - Deflection of a 3DSP for a force of $50 \mathrm{mN}$ force in $x$-, $y$ - and $z$-direction as obtained by simulations

The stiffnesses in $\mathrm{x}$ - and $\mathrm{y}$-directions are more than 1000 times as high as the stiffness in z-direction. Therefore we can assume that deflection of the SP in $\mathrm{X}$ - or $\mathrm{y}$-direction for a specified force is negligible compared to the deflection in $\mathrm{z}$-direction. With this result a necessary condition for the concept of a 3D combination of the SP is fulfilled. Further it shows that a stiffness under $1 \mathrm{mN} . \mu \mathrm{m}^{-1}$ in measurement direction $(\mathrm{z})$ is achievable.

In order to more easily compare the influence of the geometric parameters, the simulation results for each parameter were fitted with the function $y=a \cdot x^{b}$, where $y$ represents; $\mathrm{S}_{\mathrm{x}}, \mathrm{S}_{\mathrm{y}}$ or $\mathrm{S}_{\mathrm{z}}$ and $\mathrm{x} ; \mathrm{W}_{\text {mem }}, \mathrm{t}_{\text {mem }}$ or $\mathrm{L}_{\text {mem }}$. In Table 1, the fit results $a, b$ and $R^{2}$ (coefficient of determination) are given for each parameter. They were obtained through a linear regression of the natural logarithm of y. It's obvious from the results that for the tailoring of the stiffness $S_{z}$, the thickness of the membrane $t_{\text {mem }}$ can be varied. Also the length of the membrane $L_{m e m}$ could be used by paying attention to not affect too much the ratio $S_{x}-S_{y} / S_{z}$. The width of the membrane $W_{\text {mem }}$ has a bigger influence on $S_{y}(b=2.37)$ than on $S_{z}$ (linear). This parameter has therefore to be maximized to assure the maximum stiffness in $\mathrm{S}_{\mathrm{y}}$ (in practice $3.8 \mathrm{~mm}$ was later chosen).

Table 1 - Fit parameters as obtained by simulations for the study of the dimension of the hinge geometry

Influence of $\mathbf{L}_{\text {mem }}$ for $\mathrm{t}_{\mathrm{mem}}=25 \mu \mathrm{m}$ and $\mathrm{W}_{\mathrm{mem}}=3.8 \mathrm{~mm}$

\begin{tabular}{cccc}
\hline & $\boldsymbol{S}_{\boldsymbol{x}}=\boldsymbol{a} \cdot \boldsymbol{L}_{\boldsymbol{m e m}}^{\boldsymbol{b}}$ & $\boldsymbol{S}_{\boldsymbol{y}}=\boldsymbol{a} \cdot \boldsymbol{L}_{\boldsymbol{m e m}}^{\boldsymbol{b}}$ & $\boldsymbol{S}_{\mathbf{z}}=\boldsymbol{a} \cdot \boldsymbol{L}_{\mathbf{m e m}}^{\boldsymbol{b}}$ \\
\hline $\mathbf{a}$ & 11.3 & 0.899 & $3.05 \cdot 10^{-4}$ \\
\hline $\mathbf{b}$ & -0.243 & -0.328 & -0.725 \\
\hline $\mathbf{R}^{\mathbf{2}}$ & 0.997 & 0.999 & 1.00
\end{tabular}

Influence of $\mathbf{t}_{\mathbf{m e m}}$ for $\mathrm{L}_{\mathrm{mem}}=3.3 \mathrm{~mm}$ and $\mathrm{W}_{\mathrm{mem}}=3.8 \mathrm{~mm}$

\begin{tabular}{cccc}
\hline & $\boldsymbol{S}_{\boldsymbol{x}}=\boldsymbol{a} \cdot \boldsymbol{t}_{\boldsymbol{m e m}}^{\boldsymbol{b}}$ & $\boldsymbol{S}_{\boldsymbol{y}}=\boldsymbol{a} \cdot \boldsymbol{t}_{\boldsymbol{m e m}}^{\boldsymbol{b}}$ & $\boldsymbol{S}_{\boldsymbol{z}}=\boldsymbol{a} \cdot \boldsymbol{t}_{\boldsymbol{m e m}}^{\boldsymbol{b}}$ \\
\hline $\mathbf{a}$ & 102 & 11.3 & 29.8 \\
\hline $\mathbf{b}$ & 0.526 & 0.593 & 2.91 \\
\hline $\mathbf{R}^{\mathbf{2}}$ & 1.00 & 1.00 & 1.00
\end{tabular}

Influence of $\mathbf{W}_{\text {mem }}$ for $\mathrm{L}_{\mathrm{mem}}=300 \mu \mathrm{m}$ and $\mathrm{t}_{\mathrm{mem}}=25 \mu \mathrm{m}$

\begin{tabular}{cccc}
\hline & $\boldsymbol{S}_{\boldsymbol{x}}=\boldsymbol{a} \cdot \boldsymbol{W}_{\boldsymbol{m e m}}^{\boldsymbol{b}}$ & $\boldsymbol{S}_{\boldsymbol{y}}=\boldsymbol{a} \cdot \boldsymbol{W}_{\text {mem }}^{\boldsymbol{b}}$ & $\boldsymbol{S}_{\boldsymbol{z}}=\boldsymbol{a} \cdot \boldsymbol{W}_{\mathbf{m e m}}^{\boldsymbol{b}}$ \\
\hline $\mathbf{a}$ & 5.06 & $5.40 \cdot 10^{-2}$ & $1.72 \cdot 10^{-4}$ \\
\hline $\mathbf{b}$ & 0.799 & 2.37 & 1.00 \\
\hline $\mathbf{R}^{\mathbf{2}}$ & 0.998 & 0.999 & 1.00 \\
\hline
\end{tabular}

\section{FABRICATION PROCESS}

The single SP is composed of three silicon parts: the bottom spring, a spacer and the top spring (Figure 3). For the top and the bottom parts a $360 \mu \mathrm{m}$ thick (100) silicon $\mathrm{n}$-doped wafer was used and a $1 \mathrm{~mm}$ thick wafer for the spacer, which results in a total thickness of $1.72 \mathrm{~mm}$. The bottom and top spring comprise two elastic hinges made of a thin silicon membrane, which were obtained by wet etching. On the top spring piezo-resistive paths were provided by local diffusion doping within the membrane area. They were connected and wired to form a Wheatstone bridge (Figure 3). Longitudinal and also transversal orientations of the resistors were fabricated and tested. A more detailed process description can be found elsewhere [2, 6]. To structure the spacer wet etching was applied to both sides. This etching was realized in a bath of $\mathrm{KOH}$ by $80^{\circ} \mathrm{C}$. For this a hard mask made out of a thermal $420 \mathrm{~nm} \mathrm{SiO}$ and of a $300 \mathrm{~nm} \mathrm{Si}_{2} \mathrm{~N}_{3}$ layers is needed.

The three different wafers were processed separately and finally bonded together with an adhesive technique. For this bonding, a special transfer technique was used, in order to deposit glue only on surfaces to bond. In Figure 5, the adhesive bonding steps are illustrated. In a 
first step, a polyimide foil was coated with epoxy glue (353ND, Co. Epotek) by a spin coating process $\left(4000 \mathrm{~min}^{-1}, 30^{\prime}\right)$, spreading it over the foil with a constant thickness. The glue was than applied on the surface of the spacer. After peeling off the foil, a thin glue layer remained. The top and the spacer were aligned to each other with the aid of alignment marks on an alignment system (EVG®620, EVG®420 from EV Group). Finally, the glue was cured by $150^{\circ} \mathrm{C}$ during $1 \mathrm{~min}$. Then this glue transfer and alignment process was repeated for the bottom spring and the top spring/spacer stack.

Singularisation of the SPs by wafer cutting was found to create problems. By sawing silicon, the upper and lower edges of the silicon are splintered. When the saw reaches the membrane, this splintering can cause cracks. To avoid that, a retraction of the membrane is structured on wafer-level with the Bosch process (see Figure 3). In this way, a well-defined width of the membrane and a frame of thicker silicon that protects the membrane during sawing and handling are provided.

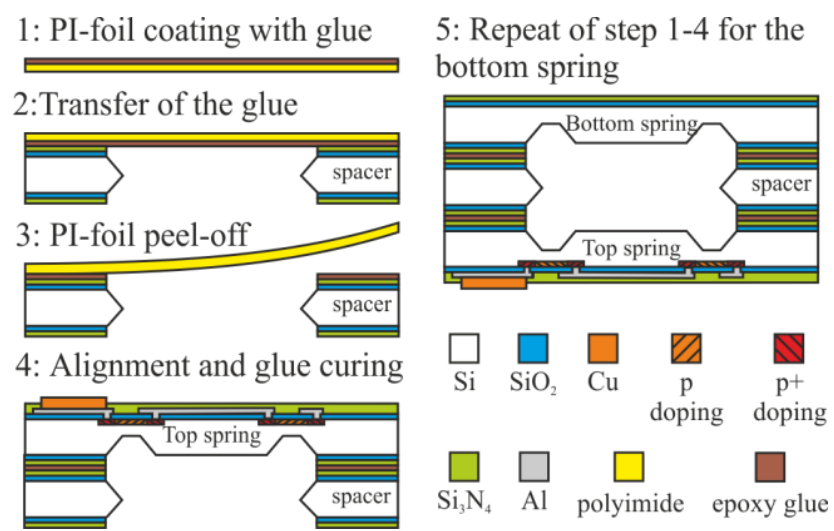

Figure 5 - On wafer-level adhesive bonding process with glue transfer

\section{EXPERIMENTAL RESULTS}

To characterize the produced SPs, they were glued to a PCB and contacted by soldering to attach them in an existent force/displacement measurement setup (Figure 6a.). For the analysis of the sensors, a linear stage M511 from PI GmbH controlled by a LabVIEW program was used to deflect the single SP [2]. To be able to characterize its mechanical behavior, a force gauge KD78 from Messtechnik $\mathrm{GmbH}$ was used. During the stepwise deflection of the SP, the position of the stage, the tension of the force gauge and the sensor tension were recorded. The tensions were acquired with the help of a data acquisition board (PCI 6289) from NI and a DAQPBRIDGE-B strain gage signal conditioning modules from Dewetron, Inc.. From the recorded data, the force, the signal response and the deflection of the SP were extracted for further analysis. Also a first prototype of a 3D microprobe made out of three identical SP was assembled and tested (Figure 6b.). For this first prototype, up to now only a mechanical characterization was carried out. To connect the SPs to the measurement setup, concepts are developed, but still were not tested. The assembly occurred manually with the help of epoxy glue and positioning equipment, to assure the orthogonality between each SP.

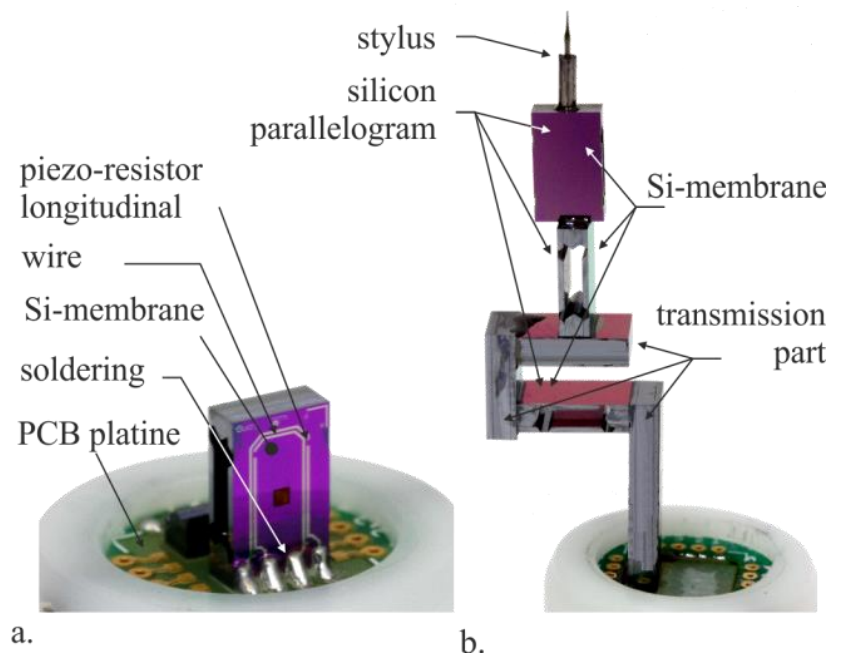

Figure 6 - a. single SP with longitudinal piezo-resistors and $b$. assembled micro parallelograms for $3 D$ micro probing

In Figure 7, both the force and the sensor response are presented in function of the deflection of the SP. The measured force behavior confirms the simulation results. However $S_{z}$ is only about 300 times smaller than $S_{x}$ and $S_{y}$, and not 1000 times as expected from simulations. The small stiffnesses $S_{x}, S_{y}$ can be explained because of the limited precision of the gauge. At a measured force of $100 \mathrm{mN}$ the deflections of the SP should be about $10 \mathrm{~nm}$ in accordance to the simulations. An important result is the proof of perfect linearity of the mechanical behavior without any hysteresis (forward and backward curves are superposed for all directions).

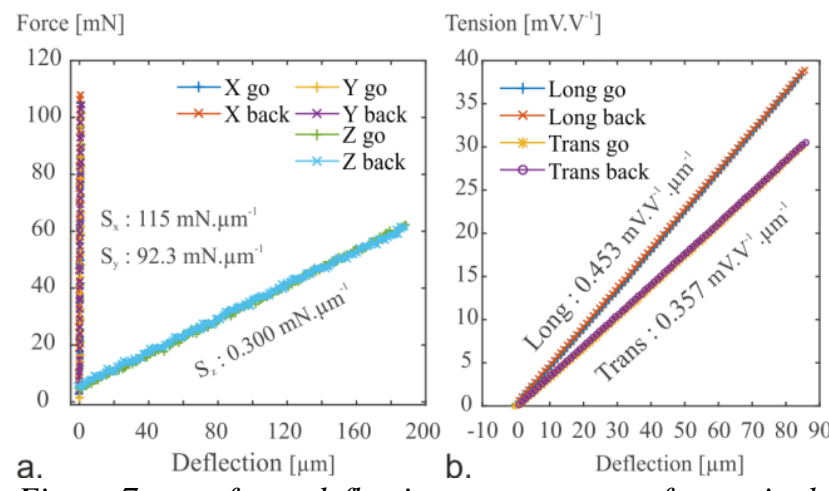

Figure 7 - a. force-deflection measurement for a single $S P$ in $x$-, $y$ - and z-direction and $b$. signal-deflection measurement for longitudinal and transversal piezoresistor arrangements

The mechanical characterization was carried out with three different membrane lengths at a membrane thickness of $26 \mu \mathrm{m}$. In Table 2, the simulated and measured values of $S_{z}$ are shown. In the studied range, the measured values are consistently 2.4 times lower than the simulated ones. At the moment we would explain this by not being able to manufacture membranes with precisely targeted thickness, which influences the stiffness with $t_{\text {mem }}{ }^{3}$ (see Table 1). 
Table 2- Simulated and measured stiffnesses together with the corresponding deflection in z-direction leading to fracture for single SP with a membrane thickness of $26 \mu \mathrm{m}$ and different membrane lengths

\begin{tabular}{lccc}
\hline $\begin{array}{l}\text { Membrane length } \mathbf{L}_{\text {mem }} \\
\text { in } \mathbf{m m}\end{array}$ & $\mathbf{0 . 4 5}$ & $\mathbf{0 . 3 5}$ & $\mathbf{0 . 2 5}$ \\
\hline Simulated $\mathbf{S}_{\mathbf{z}}$ in $\mathbf{m N} . \boldsymbol{\mu m}^{-1}$ & 0.61 & 0.73 & 0.94 \\
\hline Measured $\mathbf{S}_{\mathbf{z}}$ in $\mathbf{m N} . \boldsymbol{\mu m ^ { - 1 }}$ & $\begin{array}{c}0.26 \\
\pm 0.01\end{array}$ & $\begin{array}{c}0.31 \\
\pm 0.01\end{array}$ & $\begin{array}{c}0.38 \\
\pm 0.01\end{array}$ \\
\hline Fracture deflection in $\boldsymbol{\mu m}$ & $\begin{array}{c}713 \\
\pm 65\end{array}$ & $\begin{array}{c}548 \\
\pm 65\end{array}$ & $\begin{array}{c}488 \\
\pm 100\end{array}$ \\
\hline
\end{tabular}

The sensing characteristics for longitudinal and transversal piezo-resistor arrangements at z-direction deflection are given in Figure $7 \mathrm{~b}$. The obtained sensitivities are sufficient to enable sub-micron precision measurements. The difference between both can be explained by the distribution of the strains in the membrane. Indeed the longitudinal arrangement profits from bigger strains values, which are located on the border of the membrane (towards the outside of the SP). To optimize the sensitivities, the resistors have to be placed accordingly.

Table 2 gives also the operation ranges for SP (maximum deflection before fracture) for different membrane length with a thickness of $26 \mu \mathrm{m}$. For this experiment, eight probes were deflected until fracture and the mean values and the interval of confidence by $99.9 \%$ were determined. The use of these SP for a 3D microprobe would improve the measurement range (more than 3 times) against actual silicon microprobes.

Finally, the mechanical characterization of a 3D micro probing system (Figure 6a.) happened with and without stylus. In Table 3, the measured stiffnesses in $\mathrm{X}-$, $y$ - and z-directions are presented. Without the stylus the constructed system is perfectly isotropic. The influence of the stylus (mounted in z-direction) can be seen on $\mathrm{x}$ - and y-directions, it's bending directions. However, in both cases the maximal anisotropy was 1.2, which is lower than every microprobe of today.

Table 3 - Measured stiffness in $x$-, $y$ - and z-direction for a $3 D$ micro probing system without and with stylus constructed from three SP with a membrane thickness of $26 \mu \mathrm{m}$ and a membrane length of $0.35 \mathrm{~mm}$

\begin{tabular}{lcc}
\hline & without stylus & with stylus \\
\hline $\mathrm{S}_{\mathrm{x}}$ in $\mathbf{m N} . \mu \mathbf{m}^{-1}$ & 0.300 & 0.300 \\
\hline $\mathrm{S}_{\mathrm{y}}$ in $\mathbf{m N} . \mu \mathbf{m}^{-1}$ & 0.290 & 0.270 \\
\hline $\mathbf{S}_{\mathrm{z}}$ in $\mathbf{m N} . \mu \mathbf{m}^{-1}$ & 0.280 & 0.250 \\
\hline max. anisotropy & 1.06 & 1.20 \\
\hline
\end{tabular}

\section{SUMMARY AND OUTLOOK}

A new tactile sensor design composed of single silicon parallelograms was presented which presents numerous advantages for it use in conventional CMM and for many others application in 1D or 3D setups.

The simulations confirmed an isotropic behavior of this new design. It was also possible to optimize the geometrical parameters based on a parametric study. The developed on wafer-level fabrication enabled a low-cost realization of single SP. The mechanical and sensor response characterizations showed that over a wide deflection range $\pm 400 \mu \mathrm{m}$, a precise deflection measurement at a low stiffness was possible. The first characterization of a 3D micro probing system (without sensors) confirmed that an isotropic mechanical behavior can be achieved.

The described results prove that a new 3D microprobe with many advantages (isotropy, low stiffness and wide range) can be successfully realized. The fabrication of SP and the 3D assembly of SPs for complete measuring systems will further be optimized in near future. Concepts for getting all SPs electrically connected without mechanical interference will be tested soon.

\section{ACKNOWLEDGEMENTS}

We thank M. David for assistance during the development, fabrication and measurements.

This research was supported by the Deutsche Forschungsgemeinschaft, DFG, project number DI 1934/5-1.

\section{REFERENCES}

[1] E. J. Bos, Tactile 3D probing system for measuring MEMS with nanometer uncertainty:aspects of probing, design, manufacturing and assembly, Technische Universiteit Eindhoven, 2008.

[2] N. Ferreira, A. Brennecke, A. Dietzel, S. Buttgenbach, T. Krah, D. Metz, K. Kniel, F. Hartig, "Reducing the probe ball diameters of 3D siliconbased microprobes for dimensional metrology," 2013 Seventh International Conference on Sensing Technology (ICST), Wellington, New Zealand.

[3] S. Bütefisch, S. Büttgenbach, T. Kleine-Besten, U. Brand, "Micromechanical three-axial tactile force sensor for micromaterial characterisation," Microsystem Technologies, vol. 7, no. 4, pp. 171-74, 2001.

[4] E. Bos, R. Heldens, F. Delbressine, P. Schellekens, A. Dietzel, "Compensation of the anisotropic behavior of single crystalline silicon in a 3D tactile sensor," Sensors and Actuators A: Physical, vol. 134, no. 2, pp. 374-81, 2007.

[5] S. Buetefisch, G. Dai, H.-U. Danzebrink, L. Koenders, F. Solzbacher, M. P. Orthner, "Novel design for an ultra high precision 3D micro probe for CMM applications," Procedia Engineering, vol. 5, pp. 705-12, 2010.

[6] A. Phataralaoha, Entwicklung piezoresistiver taktiler Sensoren für die Charakterisierung von Mikrokomponenten, Shaker, Aachen, 2009.

\section{CONTACT}

*D. Metz, tel.: +49 531391 9743; d.metz@tu-bs.de 